\title{
Study on the Optimal Application of Mathematical Model to Transportation Problem
}

\author{
Weizhu Wang \\ Liaoning University of International Business and Economics, \\ Dalian 116052, Liaoning
}

\begin{abstract}
Many factors such as value, bulk, shape, and measuring unit are involved in the transportation industry and also present different transportation characteristics and the hierarchical characteristics based on the transport demands. Thus, differences in the presentation of these aspects such as transportation cost, time, frequency and security are caused. With the rapid development of the social economy and the information-based improvement of the integrated transportation industry, the integrated transport industry of cargo is developing toward the direction of diversification. In this paper, the related optimal mathematical model is proposed and constructed based on the optimization theory of multi-cargo integrated transportation organizational form, thus solving the process of the optimal model construction. It is expected to reduce the integrated cost of transportation and maximize the overall cargo transportation benefits.
\end{abstract}

Keywords- Multi-cargo Integrated Transportation; Optimal Model; Construction; Mathematical Algorithm

\section{Introduction}

Transportation problems involve every aspect, and the cargo integrated transportation organizational form is necessarily taken into consideration for the development of the national logistics industry. It covers the reasonable combination of different cargos with different transportation modes and paths. The ultimate purpose is to complete the cargo transportation tasks by giving priority to the integrated benefit and then achieve the minimum cost consumption and the maximum profits in cargo transportation.
2. The related theories for the optimization of multi-cargo integrated transportation organizational form

2.1 Theory of the interaction of the transportation organizational form optimization with its network distribution

In the optimization of the cargo integrated transportation organizational form, the key lies in choosing and dynamically integrating different transportation ways and also selecting the optimal transport path. This is a transportation solution taken into consideration based on the comprehensive benefit optimization.

Cargo transportation network flow distribution is related to not only the types of cargos and also the cargo transportation modes and paths. On the one hand, it decides the final transportation scheme and path decision and provides a reliable practical basis for the secured cargo transportation. On the other hand, it can provide feedback about the efficiency of the cargo integrated transportation scheme and also reflect the advantages and disadvantages of the running status of cargos.

2.2 Choosing the way of optimizing the multi-cargo integrated transportation organizational form

The optimization of multi-cargo integrated transportation organizational form is actually to build a network flow-distribution model and also let it abide by the specific nonlinear planning category. At this point, an integrated transportation network equilibrium and optimization model was established in this paper 
based on the broad sense of transportation costs. This model could reflect the different technical and economic characteristics and transportation time according to the different transportation characteristics and ways, and also implement the volume distribution in the actual transportation process. It is important to note that the results produced in this volume distribution process were comprehensively evaluated based on many factors such as transportation cost, time, and types, and then the rationality in the volume distribution process could be objectively reflected. Therefore, the optimization model established in this paper was determined based on the principle of the minimum transport cost, and also many elements possible in the transportation were taken into account [1].

\section{The construction of the optimal mathematical model for the multi-cargo integrated transportation organizational form}

The optimal mathematical model, mentioned in this paper, was an integrated transportation network balance flow-distribution mathematical model in a broad sense, which was constructed based on the multi-cargo integrated transportation organizational form and targeted at the optimization of the best transportation system operation.

\subsection{The definition of the relevant variables and parameters of the mathematical optimization model}

To define variables and parameters was to better describe and construct the mathematical optimization model. In this paper, $\mathrm{G}=(\mathrm{N}, \mathrm{A})$ was seen as the set of the nodes in multi-cargo integrated transportation network: $\mathrm{N}$ was the concrete sign to express this set of nodes; A was the set of united arcs; $\alpha \in A$ was the transportation arc in the multi-cargo integrated transportation network. If the transport arc $\bar{A}$ and the reloading arc $\bar{A}^{\prime}$ had a

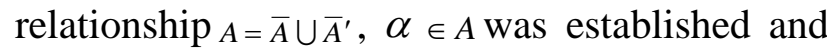

also $C_{\alpha}$ was used for expressing the integrated transportation capacity of the $\operatorname{arc} \alpha$. In the model, $\mathrm{S}$ was used for expressing the set of the ending points of the cargo transportation flow, if $R$ was the set (OD) of the starting points of all the cargo transportation flows and also $R \in N$ was established. If $S \in N, R S$ formed a set of all the cargo OD flow in the transportation network. Here, rs was assumed to be the OD flow of the determined cargo, and $r s \in R S$ was available.

In terms of multi-cargo integrated transportation categories, the set of the cargos was assumed to be $\mathrm{P}$, and then $p \in P$ was available if some type of cargos was p. In respect of transportation path, the set of optional paths was $K_{r s}^{P}$ for expressing all the paths available for the OD flow of $p$ in $r s$, and thus, $k_{r s}^{p} \in K_{r s}^{p}$ was established.

\subsection{The construction of the mathematical optimization model}

The construction of a multi-cargo integrated transportation model mainly included three parts: objective function, constraint conditions, and mathematical model.

\subsubsection{The determination of objective function}

If transport behavior as well as cost emerged because cargo was on the integrated transportation network, the differences of cargo types, transportation modes and costs were necessarily taken into account. Overall, a systematic, corresponding optimization targeted at the optimal path and minimum cost of transportation was a must, so as to achieve the optimal transportation configuration of the cargo OD flow in a broad sense.

\subsubsection{Constraint conditions}

The transportation party was required to raise a related optimization model according to the regional comprehensive transportation network characteristics, so as to give the maximum play to the superiority of the selected transport lines. 


\subsubsection{The construction of the mathematical model}

In the process of assuming the objective function and the constraint condition, the mathematical model was constructed for the optimization of the multi-cargo integrated transportation organizational form after the variable parameters were constructed and the basic instructions were implemented. The basic optimization model is as follows.

$$
\min C=\sum_{p \in P} \sum_{r \in R} \sum_{s \in S} \sum_{k \in K} f_{k}^{p, r s} C_{k}^{p, r s}
$$

Based on the optimization model, the following function model was deduced.

$$
\begin{gathered}
\sum_{p \in P} \sum_{r \in R} \sum_{s \in S} \sum_{k \in K} f_{k}^{p, r s} \delta_{\alpha, k}^{p, r s} \leq C_{\alpha}, \forall \alpha \in A \\
\sum_{k \in K} f_{k}^{p, r s}=q_{r s}^{p}, \forall_{r} \in R, s \in S, p \in P \\
f_{k}^{p, r s} \geq 0, \forall_{r} \in R, s \in S, p \in P, k \in K \\
\delta_{a, k}^{p, r s} \in\{0,1\}, \forall_{r} \in R, s \in S, p \in P, k \in K, \alpha \in A
\end{gathered}
$$

In the above mentioned models, the originally described mathematical model was the objective function, which represented the volume of freight traffic corresponding to the OD point in condition of the minimum transportation cost in a broad range under the multi-cargo integrated transportation optimization organizational form. Other models were its constraint conditions and represented in turn the capacity constraint of cargo transportation arc, the constraints of the cargo OD on transportation path and flow, the nonnegative constraints of the cargo OD on path flows, and the logical constraints between transportation paths and curves [2].

\section{Solving the optimization mathematical model for the multi-cargo integrated transportation organizational form optimization}

In this paper, the mathematical model for the multi-cargo integrated transportation optimization organizational form was solved based on the genetic algorithm of the reasonable $\mathrm{K}$ transport path, because it could objectively and really reflect the real-time state of the transported cargos in the transportation network.

\subsection{The two-way scanning algorithm of the short-path problem in $K$ optimizing transport path}

$K$ optimizing transport path referred to the minimum transport path based on the broad transportation cost. Combined with the actual situation, two or more transportation ways such as highway, railway, aviation, waterways and pipelines would be chosen and combined to form the optimal transport plan if there were multiple cargoes to be transported. This was the combination of reasonable transportation sequences. But reasonable transportation sequence was with its dynamic variability and would be relatively static within a relative time.

Also, the rationality of the transport distance was necessarily considered when transport paths were chosen. In this paper, combined with the concept of $\mathrm{K}$ optimizing transport path, the optimal transportation path was chosen from the mathematical model for the multi-cargo integrated transportation optimization organizational form.

First, two special vectors $\mathrm{k}$ were defined as dimension vectors, and thus,

$$
\bar{F}=[0, \infty, \infty, \ldots \infty], \quad \bar{V}=[\infty, \infty, \infty, \ldots, \infty]
$$

Either dimension vector was assumed to be $\mathrm{A}$, and then the following relational expression was established.

$$
\begin{aligned}
& A \Theta \bar{V}=A \\
& A \oplus \bar{V}=\bar{V} \\
& A \oplus \bar{F}=A
\end{aligned}
$$

It was assumed that the vertices Numbers were in the transportation network $(1 \sim \mathrm{n})$; if the arc coordinates were $(i, j)$, the pole was $d_{i j}$, and the following equation was obtained. 


$$
\begin{gathered}
D_{i j}=\left\lfloor d_{i j}, \infty, \infty, \ldots, \infty\right\rfloor, D=\left\lfloor D_{i j}\right\rfloor \\
\text { If } L=\left\lfloor L_{i j}\right\rfloor, \quad L_{i j}=\left\{\begin{array}{l}
\bar{V}, i \leq j \\
D_{i j}, i>j
\end{array} \quad U=\left[U_{i j}\right], \quad\right. \text { were }
\end{gathered}
$$

established, in which $U_{i j}=\left\{\begin{array}{l}D_{i j}, i<j \\ \bar{V}, i \geq j\end{array}\right.$.

In the mathematical model, $L_{i j}, D_{i j}, U_{i j}$ were the $k$-dimensional vectors and had a $k$-dimensional vector matrix (also called as a triangular matrix). If there were multiple arcs among three vertices, the following equation was obtained.

$$
D_{i j}=\left[d_{i j}^{1}, d_{i j}^{2}, \ldots, d_{i j}^{t}, \infty, \infty, \ldots, \infty\right]
$$

In the above formula, the length of the arc between $i$ and $j$ was expressed; if $\mathrm{t} \geq \mathrm{k}$, the non-existence of element $\infty$ in $D_{i j}$ was conformed. If $E_{m}^{(0)}$ was regarded as a $k$-dimensional vector, it contained the shortest path length estimate value of $\mathrm{k}$ between the original destination to the fixed point $m$, so a recurrence relational expression was solved according to $E^{(0)}$. $E^{(2 r+1)}=E^{(2 r)} \Theta E^{(2 r+1)} \oplus L, E^{(2 r+2)}=E^{(2 r+1)} \Theta E^{(2 r+2)}$

The relational expression was a basic iterative formula, in which the calculated value of $r$ was unchanged and always maintained at the optimal value in the iterative convergence if it was infinitely iterated in $0 \sim \mathrm{n}$, and then the $k$-dimensional vectors were helped to solve the shortest path of the three vertices. Analyzed from the multi-cargo integrated transportation optimization decision, cargo carrier expected to meet the differences of transportation using the alternative paths. If the optional path range was narrow, the principle was that the return path and the path needing high carrier cost were not chosen, while the shortest path and the path requiring a lower transportation cost were chosen based on the $k$-dimension vectors. Therefore, the right value of $k$ was chosen in 10\% 20\% according to actual needs; the range of $k$ value was in 3 6.

After the optimal transport path was chosen based on $k$ value, $k$ optimizing transport path was calculated according to the OD flow of the cargos. In this paper, the genetic algorithm was chosen.

\subsection{Solving $k$ optimizing transport path with the genetic algorithm}

The cores of the genetic algorithm were coding, generating initial population, designing fitness function, genetic operation, and the determination of parameter values.

\subsubsection{Chromosomal coding}

According to the encoding way of binary $0 \sim 1$, chromosomes were coded; each chromosome represented the combination of transportation path solutions of the OD flow of multiple cargoes; each gene represented an alternate transportation path. If gene $=1$, the transportation route was chosen; if gene $=0$, it was not chosen .

\subsubsection{The design of fitness function}

Based on the above chromosome code example, the objective function value of chromosomes in the optimization mathematical model was designed as $f_{1} . f_{2}, \ldots, f_{\text {pop_size }}$ to meet the fitness of the objective function. Then, a new fitness was raised according to the linear fitness scaling scheme.

$$
f_{i}^{\prime}=a f_{i}+b\left(i=1,2, \ldots, \text { pop } \_ \text {size }\right)
$$

In the formula, $f_{i}^{\prime}$ was new fitness; $a, b$ were undetermined parameters, which were chosen based on the optimal fitness. Under such a situation, the evaluation function was defined as follows:

$$
\operatorname{eval}(\mathrm{Vi})=f_{i}^{\prime} \sum_{j=1}^{\text {pop_size }} f_{j}^{\prime}, i=1,2, \ldots, \text { pop_size }
$$

Chromosomes were chosen according to the evaluation function: the probability for a chromosome to be chosen would be greater if the fitness of the chromosome was higher. Therefore, the selection of chromosomes was based on the rotating round pop_size: new chromosome was generated for the selection of a population at every time of rotation and the chromosome selection steps were as follows.

First, the cumulative probability $q_{i}$ of the chromosome $V i$ was as follows: 


$$
\left\{\begin{array}{l}
q_{0}=0 \\
q_{i}=\sum_{j=1}^{i} \operatorname{eval}\left(V_{i}\right), i=1,2, \ldots, \text { pop_size }
\end{array}\right.
$$

In the interval $\left(0, \mathrm{q}_{\text {pop_size }}\right)$, a random number $\mathrm{r}$ was produced, and then,

$$
q_{i-1}<r \leq q_{i}
$$

Then, the $i$ th chromosome $\mathrm{V}_{\mathrm{i}}$ was chosen and also met the condition of $i=1,2, \ldots$, pop_size.
By repeating the above operation, the chromosomes were replicated until the given evolution algebra and several adjacent algebras were in a non-evolutionary state and finally the optimal chromosome was output; the optimal transportation path was reasoned according to the optimal chromosome [3]. The specific operation was shown in Fig.1.

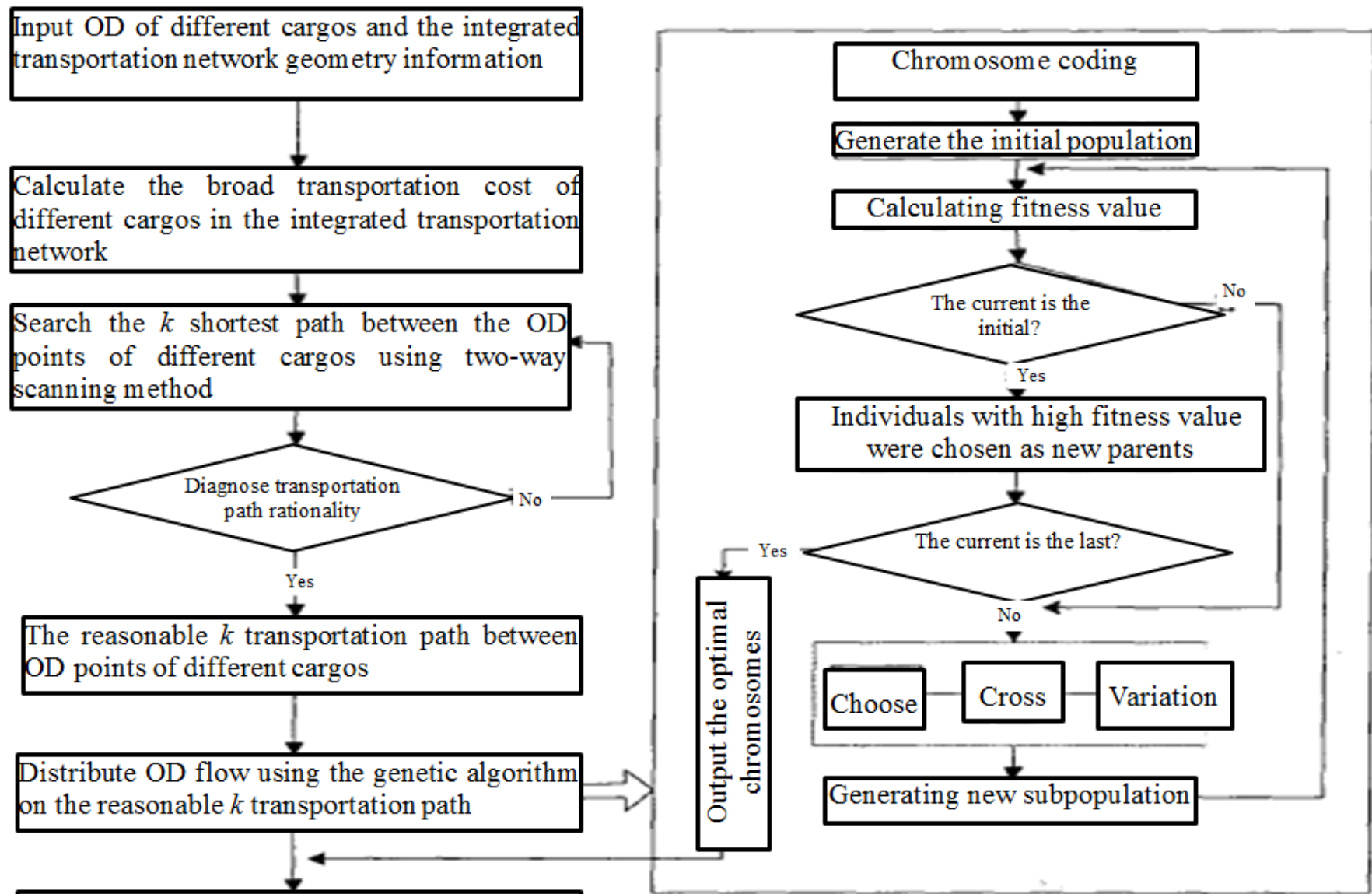

Solution for the optimization of multi-cargo integrated transportation organizational form

Fig. 1 the genetic algorithm flow of the multi-cargo transportation path optimized based on $k$ value

\section{Conclusion}

Through the analysis of the mathematical optimization model for transportation problems, a complete cargo transportation organizational optimization solution at least includes two aspects (freight volume distribution and cargo transportation path selection). In this paper, the cost minimization in a broad sense of transportation operation can be pursued only if the transportation path selection principle and mathematical optimization model mentioned in this paper were followed, and thus, the optimal transportation path was determined. 


\section{References}

[1] Xianjun Zhang. The Model and Algorithm of Uncertain Transportation Problems [D]. Chongqing University, 2006.27-31.

[2] Haijun Huang. The Optimal Mathematical Model in Balanced Transportation Network Design [J]. Journal of Aviation, 1992.13 (1).
[3] Tiexin Liu. Study on the Integrated Traffic Organizational Optimization Oriented at Complex Goods Flow [D]. Wuhan University of Technology, 2010.120-137. 